\title{
Longitudinal Changes in Performance on Cognitive Screening Tests in Patients with Mild Cognitive Impairment and Alzheimer Disease
}

\author{
Fangzhou Li ${ }^{a}$ Hajime Takechia, b Atsuko Kokuryu ${ }^{a}$ Ryosuke Takahashia \\ a Department of Neurology, Kyoto University Graduate School of Medicine, Kyoto, Japan; \\ ${ }^{b}$ Department of Geriatrics and Cognitive Disorders, Fujita Health University School of \\ Medicine, Toyoake, Japan
}

\section{Keywords}

Dementia · Memory · Executive function · Neuropsychological tests · Follow-up

\begin{abstract}
Background: Neuropsychological tests that can track changes in cognitive functions after diagnosis of Alzheimer disease (AD) and mild cognitive impairment $(\mathrm{MCI})$, including episodic memory, should be further developed. Methods: The participants of our study consisted of 22 mild AD patients and 11 MCI patients. They were followed up for 2 years. Brief cognitive screening tests were administered to the participants. Longitudinal changes in test performance were evaluated and analyzed. Results: In this longitudinal study, the Scenery Picture Memory Test (SPMT) showed significant changes over 2 years in both MCI and AD participants. The Mini-Mental State Examination (MMSE) and Word Fluency Test-vegetable showed significant changes only in AD participants. Other tests all showed little or no decline in results. Conclusions: The SPMT can be a useful tool for effectively observing changes during follow-up of MCI and AD patients.

(C) 2017 The Author(s)

Published by S. Karger AG, Basel
\end{abstract}

\section{Introduction}

Worldwide, Alzheimer disease (AD) is the most common form of dementia in elderly persons [1]. It is a neurodegenerative disorder that typically demonstrates an insidious initial impairment in memory that extends to multiple domains such as language, certain visuospatial abilities, and executive functions as the disease progresses [2-4]. Mild cognitive impairment (MCI) is a cognitive state that demonstrates measurable memory deficits between 
Li et al.: Longitudinal Changes in Performance on Cognitive Screening Tests in Patients with Mild Cognitive Impairment and Alzheimer Disease

normal aging and early dementia, and a substantial part of MCI - especially the amnestic subtype of MCI - is considered a prodrome of AD [5-7]. The early detection of dementia is important for emerging treatment possibilities and the early initiation of care, so current research has paid considerable attention to the identification of MCI and early AD.

Because episodic memory - defined as the recollection of specific past events or information - is the first and most severely affected domain in patients with $\mathrm{AD}$ and MCI [8,9], memory tests have been used in memory clinics and diagnostic centers as the first stage of screening for these conditions. Among memory tests, the Logical Memory-delayed recall subtest (LMT) of the Wechsler Memory Scale is regarded as a simple and useful screening tool with high sensitivity and specificity for differentiating normal elderly individuals from patients with AD or MCI [10-12]. Although the LMT is one of the strongest tests in predicting conversion from $\mathrm{MCI}$ to $\mathrm{AD}$, it also has a significant role in distinguishing early and late stages of MCI $[13,14]$. The Scenery Picture Memory Test (SPMT) is a recently developed memory test, and a proven and very useful tool for detecting mild and very mild AD [15].

Longitudinal studies are crucial for better understanding the progression of dementia syndromes as well as detecting the effects of pharmacological treatment and supporting the lives of patients by estimating subtle changes in performance. Little is known, however, about which psychological tests are useful for detecting changes in the cognition of patients with MCI and mild AD. Therefore, in this study, we aimed to measure the average annual rates of decline in Mini-Mental State Examination (MMSE), LMT, SPMT, and other cognitive screening test scores.

\section{Subjects and Methods}

\section{Participants}

We recruited a group of 33 patients consisting of mild AD patients $(n=22)$ and MCI patients $(n=11)$, and all were followed up for 2 years. The participants were outpatient referrals to the memory clinic of the Department of Neurology at Kyoto University Hospital. All patients underwent head computed tomography or magnetic resonance imaging, as well as a battery of laboratory tests including determination of thyroid function and vitamin $\mathrm{B}_{12}$, folate, and serum calcium concentration levels. A diagnosis of AD was made according to the criteria of the 4 th edition of the Diagnostic and Statistical Manual of Mental Disorders (DSM-IV) and the National Institute of Neurological and Communicative Disorders and StrokeAlzheimer's Disease and Related Disorders Association [16]. A diagnosis of MCI was made according to the criteria by Petersen et al. [5]. LMT Alzheimer's Disease Neuroimaging Initiative (ADNI) criteria were used to judge the memory disturbance of MCI patients. The patients were initially evaluated as part of their diagnosis before treatment with cholinesterase inhibitors. An annual longitudinal follow-up was performed at 1 and 2 years. There were MCI patients who converted to AD during follow-up; we show the longitudinal cognitive test results of the MCI group according to the initial diagnosis. All patients were $>60$ years of age and were administered cholinesterase inhibitors such as donepezil, rivastigmine, or galantamine, except for 2 patients. We prescribed cholinesterase inhibitors when MCI patients requested to take them.

The exclusion criteria used in the present study were depression, dementia with Lewy bodies, cerebrovascular disease, or any other psychological disorder. This study was performed according to the ethical guidelines for biomedical research involving human subjects set by Kyoto University, and written informed consent was obtained from all participants. 


\section{Brief Cognitive Screening Tests}

Cognitive functions were assessed using the MMSE, LMT, SPMT, Clock-Drawing Test (CDT), Block Design Test (BDT) of the Wechsler Adult Intelligent Scale-Revised (WAIS-R), and Word Fluency Test-vegetable (WFT-V). The MMSE is a global cognitive test that can be used to systematically and thoroughly assess mental status. It is an 11-question measure that tests 5 areas of cognitive function: orientation, registration, attention and calculation, recall, and language. The maximum score is 30 points. A score of $\leq 23$ points is indicative of cognitive impairment [17]. The LMT-story A was only read once to the participants, and what the participants recalled was the immediate recall subtest. The delayed recall subtest was administered $\geq 30 \mathrm{~min}$ after completion of the immediate recall subtest. The SPMT is a short and simple memory test that assesses the visual memory encoded as a scenery in combination with verbal answers. Briefly, the SPMT uses a line drawing of a living room on an A4-sized piece of paper that includes 23 objects that are commonly observed in daily life. The participants were instructed to look at the picture for $1 \mathrm{~min}$ and remember the items after an original brief digit forward test (SPMT-I). Then the participants are presented the picture again, and after this second encoding period, we distracted the participants by asking them to perform the digit span test of the WAIS-R. The participants were then asked to recall the objects in the picture without a time limit (SPMT-II). The number of items recalled was the score for the SPMT. Higher scores indicate better cognitive function [15]. For the CDT, the participants were asked to draw a clock with all numbers on a blank piece of paper and indicate the time as 10 min after 11 o'clock. We used a 10-point scoring system [18]. The BDT required individuals to use 4-16 red-and white-colored blocks to replicate a design presented as an image on a piece of paper. In the WFT-V, the participants were instructed to name as many vegetables as possible in $1 \mathrm{~min}$ [19].

\section{Statistical Analyses}

All analyses were conducted using SPSS software (version 20.0; SPSS Inc., Chicago, IL, USA). All statistics were 2-tailed, and statistical significance was set at $p<0.05$. The obtained data are presented as the means \pm standard deviations for normally distributed variables. The differences between the group means of the continuous variables were analyzed using the Student $t$ test.

\section{Results}

\section{Patient Characteristics}

Table 1 shows the demographic and clinical data for the MCI and AD groups. Age and years of education did not differ between the MCI and the AD group. As expected, the mean MMSE, SPMT-I, and SPMT-II values were significantly higher in the MCI group than in the AD group $(p<0.01)$. LMT-immediate recall showed a lower score in the AD group than in the MCI group $(p<0.05)$, while no significant differences were seen in terms of the LMT-delayed recall, CDT, BDT, or WFT-V scores between the two groups.

\section{Longitudinal Changes}

As shown in Table 2, for the MCI group a significant decline was observed in SPMT-I score from the initial evaluation $(11.2 \pm 2.9)$ to the second year $(8.6 \pm 4.4)$, and in SPMT-II score from the initial evaluation $(13.4 \pm 2.8)$ to the second year $(9.2 \pm 5.4)$. Otherwise, the MMSE, LMT-immediate recall, LMT-delayed recall, CDT, BDT, and WFT-V scores all showed almost no decline from the initial evaluation to the first year, from the first year to the second year, or from the initial evaluation to the second year. 
Li et al.: Longitudinal Changes in Performance on Cognitive Screening Tests in Patients with Mild Cognitive Impairment and Alzheimer Disease

Table 1. Demographic and clinical data at the initial evaluation

\begin{tabular}{lcc}
\hline & MCI & AD \\
\hline Patients, $n$ & 11 & 22 \\
Sex (male/female), $n$ & $2 / 9$ & $9 / 13$ \\
Age, years & $73.7(5.7)$ & $76.7(6.1)$ \\
Education, years & $12.6(2.4)$ & $11.6(2.8)$ \\
MMSE score & $26.8(1.8)^{*}$ & $24.0(2.9)$ \\
LMT-immediate recall score & $5.7(3.1)^{*}$ & $3.4(2.3)$ \\
LMT-delayed recall score & $2.3(1.8)$ & $1.2(1.9)$ \\
SPMT-I score & $11.2(2.9)^{*}$ & $6.8(3.2)$ \\
SPMT-II score & $13.4(2.8)^{*}$ & $8.1(4.9)$ \\
CDT score & $9.3(1.3)$ & $9.1(1.2)$ \\
BDT score & $27.9(6.1)$ & $21.7(9.3)$ \\
WFT-V score & $11.3(3.0)$ & $10.7(2.5)$ \\
\hline
\end{tabular}

Data are presented as the mean (SD). * Significantly different between MCI and AD patients. AD, Alzheimer disease; BDT, Block Design Test; CDT, Clock-Drawing Test; LMT, Logical Memory Test; MCI, mild cognitive impairment; MMSE, Mini-Mental State Examination; SPMT, Scenery Picture Memory Test; WFT-V, Word Fluency Test-vegetable.

For the AD group, a significant decline was observed in MMSE score from the initial evaluation $(24.0 \pm 2.9)$ to the first year $(22.9 \pm 2.5)$ and from the initial evaluation $(24.0 \pm 2.9)$ to the second year $(21.9 \pm 2.2)$. In the SPMT-I score, a significant decline was observed from the initial evaluation $(6.8 \pm 3.2)$ to the second year $(4.8 \pm 3.7)$ and from the first year $(6.1 \pm 3.3)$ to the second year $(4.8 \pm 3.7)$. In the SPMT-II score, a significant decline was observed from the initial evaluation $(8.1 \pm 4.9)$ to the first year $(6.0 \pm 4.3)$, from the first year $(6.0 \pm 4.3)$ to the second year $(3.5 \pm 4.3)$, and from the initial evaluation $(8.1 \pm 4.9)$ to the second year $(3.5$ \pm 4.3 ). We also observed significant declines in WFT-V score from the first year (10.3 \pm 3.3$)$ to the second year $(8.8 \pm 3.3)$ and from the initial evaluation $(10.7 \pm 2.5)$ to the second year $(8.8 \pm 3.3)$. We did not observe any declines in LMT-immediate recall, LMT-delayed recall, CDT, or BDT scores.

\section{Test Score Scatter for the LMT and SPMT Results}

To delineate the nature of the changes in scores on the LMT and SPMT, the longitudinal changes in the scores were plotted (Fig. 1). MCI and AD are combined in this plot. As shown in this figure, the scores on the LMT were already low even at the initial time of testing. Scores of 0 were frequently observed on the LMT-delayed recall.

\section{Discussion}

In this study, we performed a longitudinal follow-up over a 2-year period of patients with MCI and AD using the MMSE, LMT, SPMT, CDT, BDT, and WFT-V. By analyzing the changes in these brief cognitive screening tests, we observed that the SPMT can detect cognitive changes more sensitively than the other tests over 2 years. However, the LMT, CDT, and BDT all showed almost no decline in scores. Specifically, LMT-immediate recall and LMT-delayed recall scores neither demonstrated an increase nor lacked a linear downward trend in either group. Although the MMSE and WFT-V could detect longitudinal changes in AD, no significant changes in MCI were observed. 
Table 2. Longitudinal changes in brief cognitive screening tests

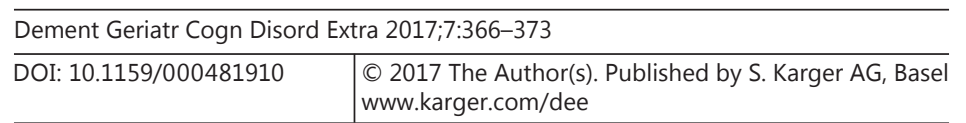

Li et al.: Longitudinal Changes in Performance on Cognitive Screening Tests in Patients with Mild Cognitive Impairment and Alzheimer Disease

\begin{tabular}{|c|c|c|}
\hline & MCI & $\mathrm{AD}$ \\
\hline \multicolumn{3}{|l|}{ MMSE score } \\
\hline Initial & $26.8(1.8)$ & $24.0(2.9)$ \\
\hline 1st year & $25.7(2.1)$ & $22.9(2.5)^{*}$ \\
\hline 2nd year & $24.8(3.8)$ & $21.9(2.2)^{\dagger \dagger}$ \\
\hline \multicolumn{3}{|c|}{ LMT-immediate recall score } \\
\hline Initial & $5.7(3.1)$ & $3.4(2.3)$ \\
\hline 1st year & $5.4(3.3)$ & $3.7(2.6)$ \\
\hline 2nd year & $4.1(4.0)$ & $3.2(2.6)$ \\
\hline \multicolumn{3}{|c|}{ LMT-delayed recall score } \\
\hline Initial & $2.3(1.8)$ & $1.2(1.9)$ \\
\hline 1st year & $2.2(2.9)$ & $1.2(1.9)$ \\
\hline 2nd year & $2.1(3.6)$ & $0.8(2.0)$ \\
\hline \multicolumn{3}{|l|}{ SPMT-I score } \\
\hline Initial & $11.2(2.9)$ & $6.8(3.2)$ \\
\hline 1st year & $9.8(3.7)$ & $6.1(3.3)$ \\
\hline 2nd year & $8.6(4.4)^{\dagger}$ & $4.8(3.7)^{\S, \dagger}$ \\
\hline \multicolumn{3}{|l|}{ SPMT-II score } \\
\hline Initial & $13.4(2.8)$ & $8.1(4.9)$ \\
\hline 1st year & $11.5(5.5)$ & $6.0(4.3)^{* *}$ \\
\hline 2nd year & $9.2(5.4)^{\dagger}$ & $3.5(4.3)^{\S \S, \dagger \dagger}$ \\
\hline \multicolumn{3}{|l|}{ CDT score } \\
\hline Initial & $9.3(1.3)$ & $9.1(1.2)$ \\
\hline 1st year & $9.4(0.8)$ & $9.0(1.2)$ \\
\hline 2nd year & $8.3(3.1)$ & $8.0(2.6)$ \\
\hline \multicolumn{3}{|l|}{ BDT score } \\
\hline Initial & $27.9(6.1)$ & 21.7 (9.3) \\
\hline 1st year & $27.6(7.1)$ & $19.9(8.9)$ \\
\hline 2nd year & $25.3(5.2)$ & $19.7(11.7)$ \\
\hline \multicolumn{3}{|l|}{ WFT-V score } \\
\hline Initial & $11.3(3.0)$ & $10.7(2.5)$ \\
\hline 1st year & $11.0(3.2)$ & $10.3(3.3)$ \\
\hline 2nd year & $10.6(3.7)$ & $8.8(3.3)^{\S, \dagger \dagger}$ \\
\hline
\end{tabular}

Data are presented as the mean (SD). $* p<0.05,{ }^{* *} p<0.01$ (initial evaluation to 1 year); ${ }^{\dagger} p<0.05,{ }^{\dagger \dagger} p<0.01$ (initial evaluation to 2 years); $\S p<0.05,{ }^{\S} p<0.01$ ( 1 year to 2 years). AD, Alzheimer disease; BDT, Block Design Test; CDT, Clock-Drawing Test; LMT, Logical Memory Test; MCI, mild cognitive impairment; MMSE, Mini-Mental State Examination; SPMT, Scenery Picture Memory Test; WFT-V, Word Fluency Test-vegetable.

These findings suggest that the SPMT is useful for judging the effects of treatment or for effectively observing longitudinal changes. This effectiveness of the SPMT may come from the "picture superiority effect" that could help memory and avoid the floor effect in patients at this stage [20-23]. The SPMT is also easy to administer, does not threaten the patient, and takes very little time. SPMT scores showed neither a floor effect nor a ceiling effect in the early stages of cognitive impairment.

As suggested in previous research, the LMT is one of the best-performing tests for discriminating between patients who are healthily aged and patients with mildly dementia $[11,12]$. AD and MCI patients fail this item because of their episodic memory deficits. As a result, the LMT can detect impairment during an early stage, but we did not see a significant decline because of the floor effect, and this test is excessively difficult to use in the very early 
Dementia

Cognitive Disorders

\begin{tabular}{l|l}
\hline DOI: $10.1159 / 000481910$ & $\begin{array}{l}\text { C } 2017 \text { The Author(s). Published by S. Karger AG, Basel } \\
\text { www.karger.com/dee }\end{array}$ \\
\hline
\end{tabular}

Li et al.: Longitudinal Changes in Performance on Cognitive Screening Tests in Patients with Mild Cognitive Impairment and Alzheimer Disease

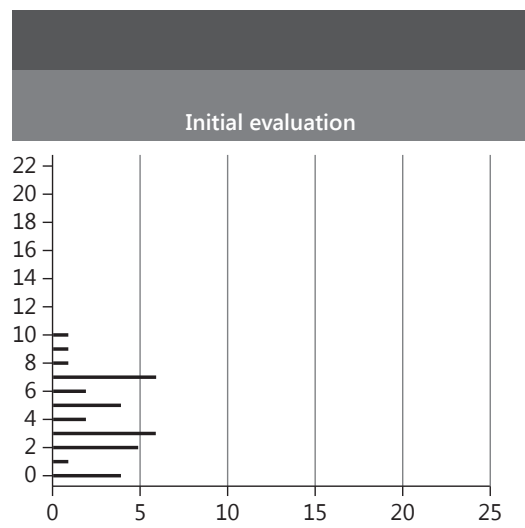

Test score scatter in LMT and SPMT

\section{LMT immediate recall} 1st year

2nd year
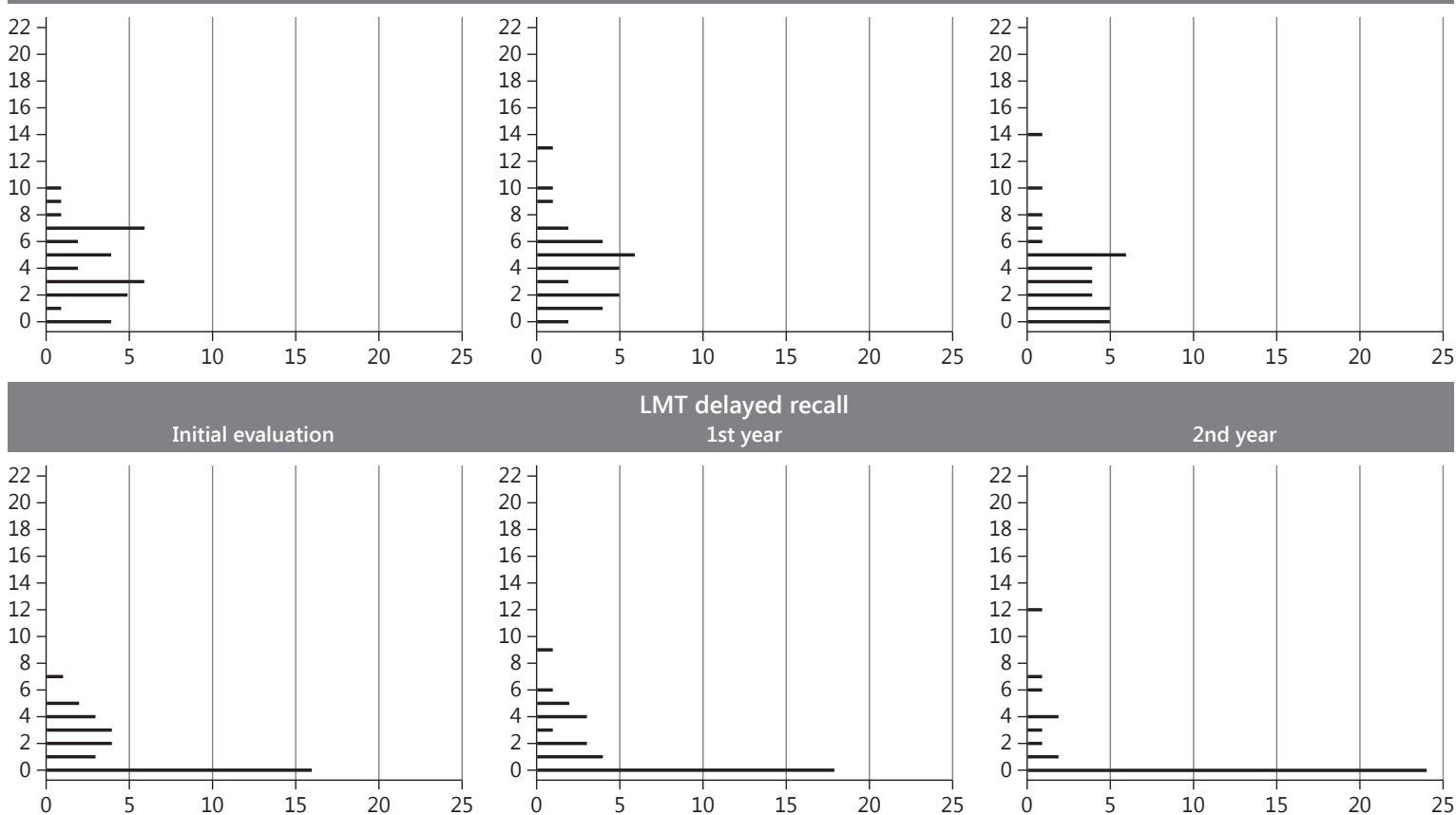

LMT delayed recall 1st year

2nd year
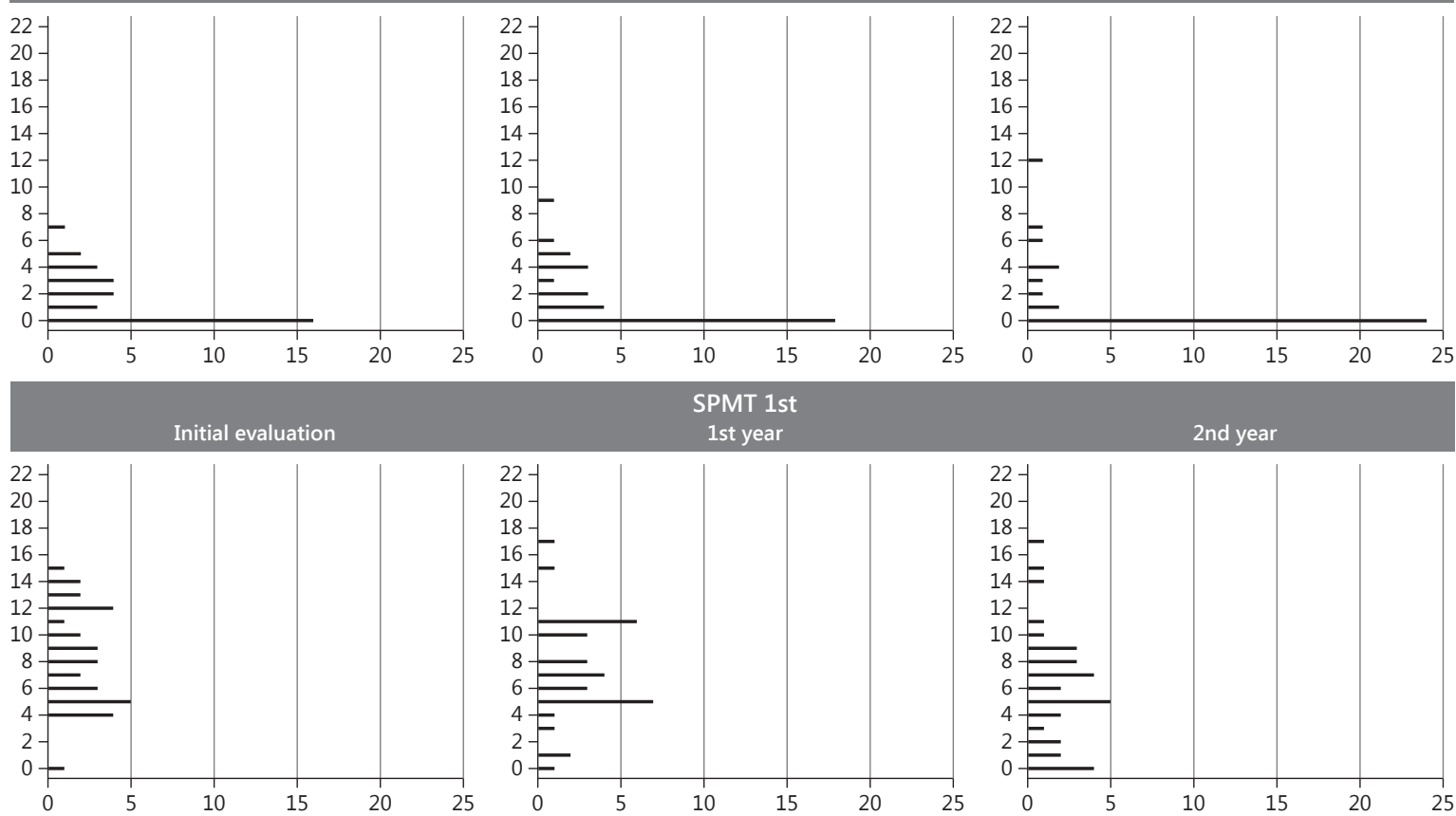

SPMT 1st 1st year 2nd year
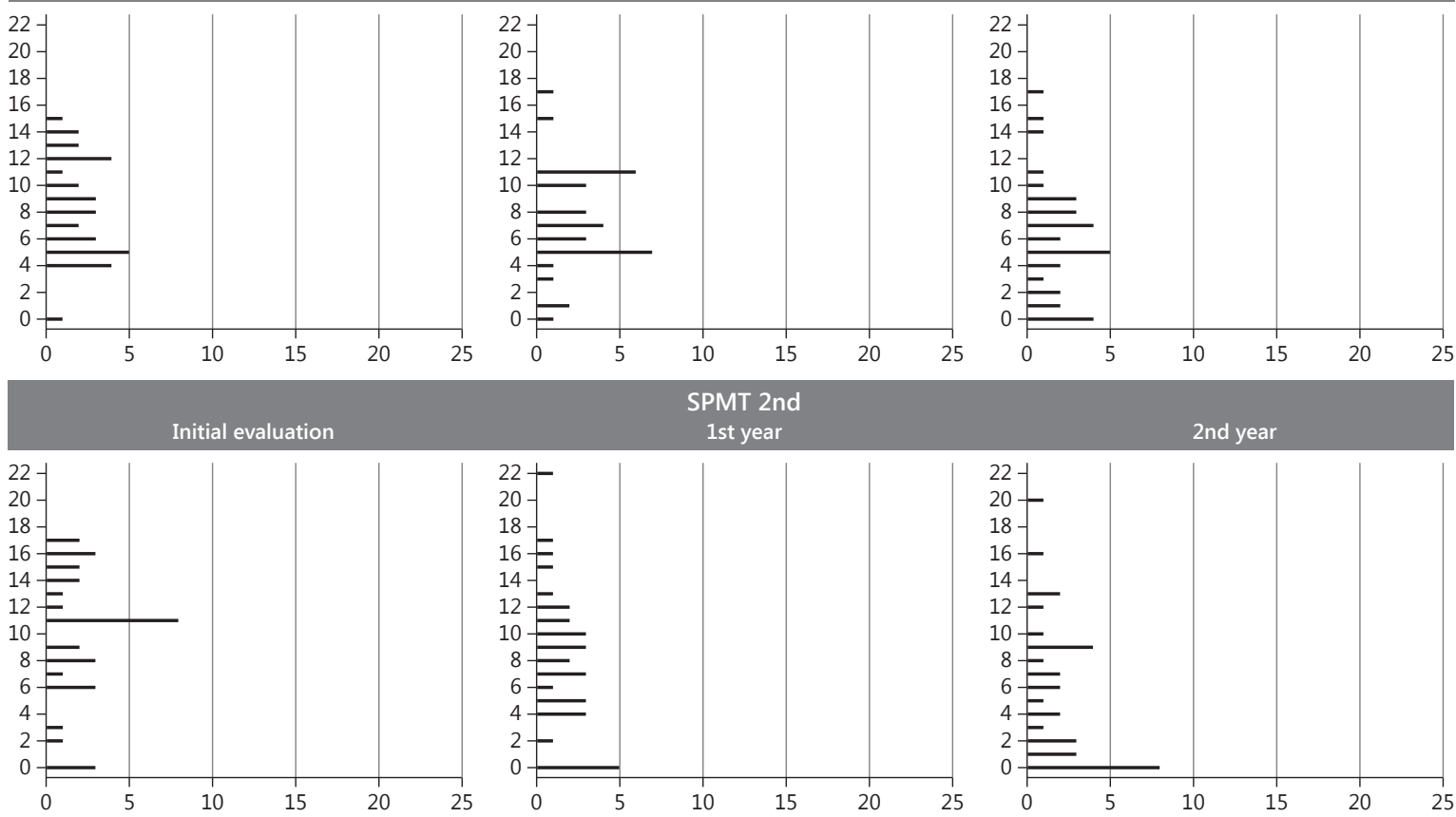

SPMT 2nd

1st year

2nd year
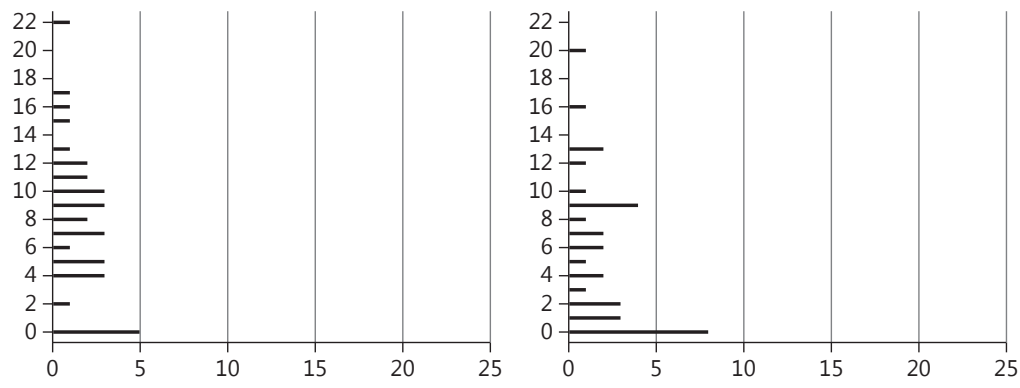

Fig. 1. Test score scatter for the LMT and SPMT. Mild cognitive impairment and Alzheimer disease are combined in this plot. LMT, Logical Memory Test; SPMT, Scenery Picture Memory Test. 
stages of dementia. Consequently, the LMT demonstrated a lack of power to detect longitudinal changes in this study.

The MMSE is a widely used screening tool for detecting and evaluating longitudinal changes in dementia patients; however, it has been suggested that it is not appropriate for determining cognitive status during the MCI phase of dementia [24]. Our results demonstrate the weak detection power of the MMSE, not only for detecting but also tracking changes in MCI. Likewise, the WFT-V is a test used to mainly assess frontal lobe function, semantic memory, and language ability, which are injured in moderate $\operatorname{AD}[25,26]$, and would demonstrate similar results to the MMSE in this study.

The CDT imposes demands on a wide range of cognitive skills, including auditory attention, semantic knowledge, planning and organization, and visuospatial functions [27]. The BDT also assesses a wide range of cognitive skills, including perceptual and visual organization, abstract thinking, nonverbal concept formation, and visuospatial function. It seems that these tests are not sensitive enough to detect longitudinal changes during the early phase of dementia.

Limitations should be considered when interpreting our results. Our proposed method determines predictions based on longitudinal data, and thus requires each participant to have the corresponding modality data across different time points, which limits the sample size. Further studies using a large sample size would be required to generalize our results. Second, we only used the LMT as the reference memory test in this study. Other potentially useful tests could be taken to determine longitudinal changes in cognition. Therefore, the generalization of our results could be limited. Third, the LMT ADNI criteria were used to judge the memory disturbance of the MCI patients in this study. One may argue that use of the LMT in the diagnosis of MCI may underestimate the role of the LMT during follow-up. However, as the distribution of LMT scores at the initial evaluation indicates, the LMT scores of the participants were apparently lower than the cutoff. This suggests that there were almost no patients who were excluded from the MCI group due to the LMT score.

In conclusion, this study shows that the SPMT is a very useful tool for longitudinal studies on MCI and mild AD. Longitudinal follow-up with the SPMT might be a useful tool for determining the effects of treatment and for effectively observing changes.

\section{Acknowledgment}

This study was supported by the Ministry of Education, Culture, Sports, Science and Technology (MEXT) of Japan (Grants-in-Aid for Scientific Research 18590598 and 22613002).

\section{Disclosure Statement}

The authors declare that they have no conflict of interest.

\section{References}

1 Ferri CP, Prince M, Brayne C, Brodaty H, Fratiglioni L, Ganguli M, Hall K, Hasegawa K, Hendrie H, Huang Y, et al: Global prevalence of dementia: a Delphi consensus study. Lancet 2005;366:2112-2117.

-2 Binetti G, Magni E, Padovani A, Cappa SF, Bianchetti A, Trabucchi M: Executive dysfunction in early Alzheimer's disease. J Neurol Neurosurg Psychiatry 1996;60:91-93.

-3 Collette F, Van der Linden M, Salmon E: Executive dysfunction in Alzheimer's disease. Cortex 1999;35:57-72.

4 Cummings JL, Cole G: Alzheimer disease. JAMA 2002;287:2335-2338. 
5 Petersen RC, Smith GE, Waring SC, Ivnik RJ, Tangalos EG, Kokmen E: Mild cognitive impairment: clinical characterization and outcome. Arch Neurol 1999;56:303-308.

-6 Petersen RC, Stevens JC, Ganguli M, Tangalos EG, Cummings JL, DeKosky ST: Practice parameter: early detection of dementia: mild cognitive impairment (an evidence-based review). Report of the Quality Standards Subcommittee of the American Academy of Neurology. Neurology 2001;56:1133-1142.

7 Ritchie K, Touchon J: Mild cognitive impairment: conceptual basis and current nosological status. Lancet 2000; 355:225-228.

-8 Li M, Ng TP, Kua EH, Ko SM: Brief informant screening test for mild cognitive impairment and early Alzheimer's disease. Dement Geriatr Cogn Disord 2006;21:392-402.

-9 Small BJ, Basun H, Bäckman L: Three-year changes in cognitive performance as a function of apolipoprotein E genotype: evidence from very old adults without dementia. Psychol Aging 1998;13:80-87.

-10 Chapman RM, Mapstone M, Gardner MN, Sandoval TC, McCrary JW, Guillily MD, Reilly LA, DeGrush E: Women have farther to fall: gender differences between normal elderly and Alzheimer's disease in verbal memory engender better detection of Alzheimer's disease in women. J Int Neuropsychol Soc 2011;17:654-662.

11 O'Caoimh R, Gao Y, Gallagher PF, Eustace J, McGlade C, Molloy DW: Which part of the Quick mild cognitive impairment screen $(\mathrm{Q} m \mathrm{mi})$ discriminates between normal cognition, mild cognitive impairment and dementia? Age Ageing 2013;42:324-330.

12 Storandt M, Hill RD: Very mild senile dementia of the Alzheimer type. II. Psychometric test performance. Arch Neurol 1989;46:383-386.

13 Doi T, Shimada H, Makizako H, Yoshida D, Shimokata H, Ito K, Washimi Y, Endo H, Suzuki T: Characteristics of cognitive function in early and late stages of amnestic mild cognitive impairment. Geriatr Gerontol Int 2013; 13:83-89.

14 Rabin LA, Paré N, Saykin AJ, Brown MJ, Wishart HA, Flashman LA, Santulli RB: Differential memory test sensitivity for diagnosing amnestic mild cognitive impairment and predicting conversion to Alzheimer's disease. Neuropsychol Dev Cogn B Aging Neuropsychol Cogn 2009;16:357-376.

15 Takechi H, Dodge HH: Scenery Picture Memory Test: a new type of quick and effective screening test to detect early stage Alzheimer's disease patients. Geriatr Gerontol Int 2010;10:183-190.

-16 McKhann G, Drachman D, Folstein M, Katzman R, Price D, Stadlan EM: Clinical diagnosis of Alzheimer's disease: report of the NINCDS-ADRDA Work Group under the auspices of Department of Health and Human Services Task Force on Alzheimer's Disease. Neurology 1984;34:939-944.

17 Folstein MF, Folstein SE, McHugh PR: "Mini-mental state." A practical method for grading the cognitive state of patients for the clinician. J Psychiatr Res 1975;12:189-198.

18 Rouleau I, Salmon DP, Butters N, Kennedy C, McGuire K: Quantitative and qualitative analyses of clock drawings in Alzheimer's and Huntington's disease. Brain Cogn 1992;18:70-87.

19 Funabiki Y, Takechi H, Akamatsu T, Kita T: Development of a short neuropsychological battery to screen early dementia in the elderly. Geriatr Gerontol Int 2002;2:179-186.

20 Lezak M, Howieson D, Loring D, Hannay J, Fischer J: Neuropsychological Assessment. 3. New York, Oxford University Press, 1995.

21 Nickerson RS: A note on long-term recognition memory for pictorial material. Psychon Sci 1968;11:58.

22 Park DC, Puglisi JT, Sovacool M: Memory for pictures, words, and spatial location in older adults: evidence for pictorial superiority. J Gerontol 1983;38:582-588.

23 Shepard RN: Recognition memory for words, sentences, and pictures. J Verbal Learn Verbal Behav 1967;6: 156-163.

-24 Chapman KR, Bing-Canar H, Alosco ML, Steinberg EG, Martin B, Chaisson C, Kowall N, Tripodis Y, Stern RA: Mini Mental State Examination and Logical Memory scores for entry into Alzheimer's disease trials. Alzheimers Res Ther 2016;8:9.

25 Hodges JR: Cognitive Assessment for Clinicians. Oxford, Oxford University Press, 2007.

-26 Isaacs B, Kennie AT: The Set test as an aid to the detection of dementia in old people. Br J Psychiatry 1973;123: 467-470.

27 Cosentino S, Jefferson A, Chute DL, Kaplan E, Libon DJ: Clock drawing errors in dementia: neuropsychological and neuroanatomical considerations. Cogn Behav Neurol 2004;17:74-84. 